\title{
Bûchers d'autrefois et guerres à venir : Valerio Evangelisti et l'inquisiteur Nicola Eymerich entre littérature populaire et discours engagé
}

\section{Éric Vial}

\section{(2) OpenEdition}

\section{Journals}

Édition électronique

URL : http://journals.openedition.org/cei/279

DOI : $10.4000 /$ cei. 279

ISSN : 2260-779X

Éditeur

UGA Éditions/Université Grenoble Alpes

\section{Édition imprimée}

Date de publication : 15 juin 2005

Pagination : 125-138

ISBN : 978-2-84310-066-6

ISSN : $1770-9571$

Référence électronique

Éric Vial, « Bûchers d'autrefois et guerres à venir : Valerio Evangelisti et l'inquisiteur Nicola Eymerich entre littérature populaire et discours engagé », Cahiers d'études italiennes [En ligne], 3 | 2005, mis en ligne le 15 décembre 2006, consulté le 27 mars 2021. URL : http://journals.openedition.org/cei/279 DOI : https://doi.org/10.4000/cei.279 


\title{
BÛCHERS D'AUTREFOIS ET GUERRES À VENIR
}

\author{
VALERIO EVANGELISTI ET L'INQUiSiTEUR NiCOLA EyMERICH \\ ENTRE LITTÉRATURE POPULAIRE ET DISCOURS ENGAGÉ
}

\author{
Éric Vial \\ Université Pierre Mendès France - Grenoble 2
}

Je crains que cet article ne souffre à la fois d'un apparent déficit de légitimité quant au sujet, et d'un autre déficit, tout à fait réel celui-là, de son auteur, qui n'est pas italianisant : enseigner l'histoire amène souvent à parler de guerres et même, de plus en plus, de leur représentation, mais cela ne donne pas automatiquement les instruments nécessaires pour en décortiquer l'aspect littéraire. Je pourrais me dissimuler derrière un rôle de traducteur, mais de quatre nouvelles de Valerio Evangelisti, ce qui est fort peu au regard des quatorze volumes publiés en dix ans, depuis $1994^{1}$ (aux-

1. Nicolas Eymerich inquisitore, Milan, Mondadori, 1994 [Nicolas Eymerich, inquisiteur, trad. Serge Quadruppani, Paris, Rivages, 1998] ; Le catene di Eymerich, Milan, Mondadori, 1995 [Les Chaînes d'Eymerich, trad. S. Quadruppani, Paris, Rivages, 1998 avec intégration de la nouvelle "Gorica » et amputation d'un chapitre] ; Il corpo e il sangue di Eymerich, Milan, Mondadori, 1996 [Le corps et le sang d'Eymerich, trad. S. Quadruppani, Paris, Rivages, 1999] ; L'ombra di Eymerich (réédition avec modification des trois volumes précédents et de la nouvelle "O Gorica tu sei maledetta »), Milan, Mondadori, $2000 ;$ Il mistero dell'inquisitore Eymerich, Milan, Mondadori, 1996 [Le Mystère de l'inquisiteur Eymerich, trad. S. Quadruppani, Paris, Rivages, 1999] ; Cherudek, Milan, Mondadori, 1997 [Cherudek, trad. S. Quadruppani, Paris, Rivages, 2000]; Picatrix, la scala per l'inferno, Milan, Mondadori, 1998 [Picatrix - L'échelle pour l'enfer, trad. Sophie Bajard, Paris, Rivages, 2002] ; I sentieri perduti di Eymerich (réédition avec modification des trois volumes précédents), Milan, Mondadori, 2000 ; Metallo urlante, Turin, Einaudi, 1998 [Métal hurlant, trad. S. Bajard, Jacques Barbéri, S. Quadruppani, É. Vial. Paris, Rivages, 2001, sans l'épilogue); Magus : Il Presagio, Milan, Mondadori 1999 [Le Roman de NostradamusLe Présage, trad. S. Bajard, Paris, Payot, 1999] ; Magus. L’inganno, Milan, Mondadori 1999 [Le Roman de Nostradamus - Le Piège, trad. S. Bajard, Paris, Payot, 1999] ; Magus : L’Abisso, Milan, Mondadori, 1999 [Le Roman de Nostradamus - L’Abîme, trad. S. Bajard, Paris, Payot, 1999] ; Magus : il Romanzo di Nostradamus (réédition des trois volumes précédents), Milan, Mondadori, 2000 ; Il castello di Eymerich, Milan, Mondadori, 2001 ; Black Flag, Turin, Einaudi, 2002 [Black flag, trad. Jacques Barbéri, Paris, Rivages, 2003] ; Mater Terribilis, Milan, Mondadori, 2002 ; Antracite, Milan, Mondadori, 2003 [Anthracite, trad. J. Barberi, Paris, Rivages, 2004] ; on ajoutera ici Gocce nere, feuilleton, Liberazione, août 2001 [comprenant les nouvelles " Il nodo Kappa " et "Sepultura " dont la seconde faisait aussi partie de Metallo urlante) et La furia di Eymerich, scénario de bande dessinée, dessins de Francesco Mattioli, Milan, Mondadori, 2003. 
quels s'ajoutent quelques nouvelles non encore intégrées dans des volumes, parfois écrites pour une publication en France avant d'être reprises en Italie $^{2}$, ainsi que des essais consacrés pour l'essentiel aux littératures dites " de genre » ou des interventions sur l'actualité ${ }^{3}$ ). Je pourrais aussi tenter de mettre en avant le fait que Valerio Evangelisti ${ }^{4}$ est lui aussi - de formation - historien, ou essayer d'annuler ma propre illégitimité par sa nonappartenance proclamée à la littérature qualifiée de "légitime ", de "blanche " ou de " générale ", c'est-à-dire par son statut revendiqué 5 d'écrivain « de genre ", ou " populaire ", d'artisan, de feuilletoniste, voire de «serial writer » renvoyant volontiers aux aventures de Sandokan racontées par Emilio Salgari, et surtout à Fantomas, ou encore au Nero Wolfe des romans policiers de l'Américain Rex Stout ${ }^{6}$. On peut invoquer ici, en particulier à propos du dernier nommé, les analyses d'Umberto Eco dans Il superuomo di massa ${ }^{7}$, mais en même temps, ce ne sont pas tout à fait les

2. "Il nodo Kappa », Valerio Evangelisti (dir.), Tutti i denti del mostro sono perfetti, Milan, Mondadori, 1998 [ "Kappa », trad. J. Barbéri : Valerio Evangelisti (dir.), Fragments d'un miroir brisé, Paris, Payot, 1999] ; participation à "Raven, jamais plus " avec Andreas Eschbach, Paul J. MacAuley, Rodolfo Martinez et Jean-Claude Dunyach, trad. É. Vial : Galaxies n ${ }^{\circ} 22,2001$; "Le souffle des FARC ", trad. É. Vial : Jean-Marc Ligny (dir.), Eros Millénium, Paris, J’ai Lu, 2001 ["Il soffio delle FArC ", Mano n 7 , 2002] ; "La fuite hors de la couveuse ", trad. S. Quadruppani : Hauteurs - Revue littéraire du Nord et d'ailleurs n 5, 2001 ; "Skinheads" (chapitre non traduit dans Les chaînes d'Eymerich), trad. Guy Pelosato, Phénix n 57, 2002 ; "Laurel \& Hardy, Terror Détectives », trad. É. Vial : Stéphane Nicot (dir.), Détectives de l'impossible, Paris, J'ai Lu, 2002 ["Stanlio \& Ollio, Terror Detectives " : F. Batini, C. Lucarelli (dir.), Anche i maschi partoriscono, Zona, 2002] ; "Caccia al cinghiale ", Il Manifesto, 15 août 2002 ; "Guerre stellari preventive ", Il Manifesto, 17 août 2003 ["Angry Red planet ", trad. É. Vial : Bruno della Chiesa (dir.), Utopiae 2003, Nantes, L'Atalante, 2003] ; "Rachid ", A. Bertante, Dieci storie per la pace, Piemme, 2003.

3. Cf. les textes de Alla periferia di Alphaville. Interventi sulla paraletteratura, Naples, L'Ancora del Mediterraneo, 2000. En langue française, $c f$. «Une littérature des étages inférieurs, la science-fiction en prise avec le monde ", Le Monde diplomatique, août 2000, repris dans Stéphane Manfredo, La sciencefiction aux frontières de l'homme, Paris, Gallimard, 2000, et dans Europe, n870, 2001 ; "L'extrême droite investit la science-fiction ", Le Monde diplomatique, octobre 2001 ; " Dans le chaos de l'après guerre pulsion de mort ", idem, mai 2003.

4. Sur Valerio Evangelisti, cf. en France, Galaxies, n ${ }^{\circ} 11$, Hiver 1998-1999, et Phénix, n 57 , mars 2002 .

5. "A quel punto, ero libero di coltivare il sogno che accarezzavo fin da bambino : diventare uno scrittore popolare. Attenzione : non un grande scrittore, e nemmeno ricco e famoso, ma uno scrittore da bancarella, da edicola della stazione. Qualcuno capace di proiettare la gente in mondi insoliti, misteriosi o fantastici, magari solo per il tempo di un viaggio in treno o di una sosta in albergo. La meta è ancora lontana, ma mi sono avvicinato parecchio ». Interview de Valerio Evangelisti, L'Unità, 16 avril 1996.

6. "L'imitazione di Nero Wolfe venne molto più tardi, quando decisi di dedicarmi alla narrativa popolare trasfondendovi un poco di tutto ciò che avevo amato leggere in gioventù. Il mio eroe sarebbe stato anche lui asociale e misantropo (molto, molto di più) ; anche lui avrebbe avuto comportamenti ricorrenti e maniacali, che il lettore si sarebbe divertito a cercare e ritrovare in ogni romanzo. Non voglio dire che il mio Eymerich sia stato ricalcato su Nero Wolfe, ma certo ne replica taluni aspetti ; soprattutto, il ciclo che lo vede protagonista applica i meccanismi di assuefazione che avevo scoperto nei romanzi di Rex Stout, e di cui Umberto Eco mi aveva fornito la chiave strutturale... » Valerio Evangelisti, « Eymerich deve tanto a Nero Wolfe ", Letture, juin-juillet 1999. Cf. également Valerio Evangelisti, "L'ideologia di Nero Wolfe ", 1997, rééd. in Alla periferia... op. cit.

7. Milan, Bompiani, 1978. Traduction partielle, De Superman au surhomme, Paris, Grasset, 1993. 
catégories où l'on rangera Antonio Tabucchi ou Carlo Sgorlon, Pier Paolo Pasolini ou Leonardo Sciascia. Tant pis, ou tant mieux, si cela permet de s'interroger sur les convergences ou les divergences d'approche de la violence et de la guerre dans un canton de la littérature populaire italienne et dans la littérature « légitime». D'autant que, peut-être à titre de provocation, Valerio Evangelisti est prompt à proclamer que cette dernière est enfermée dans un monde artificiel ou passé, incapable de rendre compte des réalités présentes, contrairement aux genres " des étages inférieurs ${ }^{8}$ ". Ce qui pourra être vérifié ou infirmé dans le cas des violences et des guerres.

Celles-ci sont, de fait, fort présentes chez Evangelisti, sans qu'il s'agisse d'une conséquence inéluctable du genre de littérature qu'il a choisi. En effet, à l'évidence, d'autres formes porteuses de suspense sont tout à fait possibles : on les rencontre de fait dans ses textes, et elles pourraient suffire à faire avancer et fonctionner le récit. C'est au premier chef le cas de l'enquête, que l'enquêteur soit confronté au merveilleux, au fantastique, à l'impossible, à l'insolite, dans la tradition des "détectives de l'étrange ", à des intrigues et combinaisons politiques, ou encore à des faits délictueux supposant certes une violence, mais antérieure au récit. On ajoutera que le héros récurrent, central dans huit des volumes publiés ${ }^{9}$, et dominant dans le neuvième ${ }^{10}$, Nicola Eymerich (personnage historique réel, né en 1320,

8. "Je crois qu’aujourd'hui la littérature blanche, la littérature générale, a renoncé complètement, au moins en Italie, à s'interroger sur le monde où on vit. Si on regarde froidement ce monde, on voit par exemple un continent entier, l'Afrique, qui est mort, complètement déchiré par des guerres sauvages dont on ne comprend même plus l'origine; il y a aussi des choses comme ça en Asie, et dans une partie au moins de l'Amérique latine. Mais l'écrivain typique de littérature blanche s'en fout complètement [...]. En réalité, ce sont eux qui font de la littérature fantastique, ils sont complètement détachés de la réalité, et pas seulement sur le plan politique mais aussi sur celui de la vie quotidienne : il est difficile de parler de la réalité actuelle sans voir ce qu'Internet représente, ou de parler de ce qui nous entoure sans dire que les ordinateurs ont changé beaucoup de choses, à commencer par la façon de travailler, et tout ça, et bien le lettré typique, l'homme de la haute culture, s'en fout complètement. Quelquefois il revendique son hostilité à l'ordinateur, il dit qu'il écrit à la plume... Oui, il écrit à la plume, mais la majorité des gens autour de lui travaillent avec l'ordinateur, donc de quelle réalité est-il en train de nous parler ? Moi qui suis un écrivain de l'imaginaire, je parle d'une réalité beaucoup plus réelle que la sienne ". Interview de Valerio Evangelisti dans Galaxies, revue citée. "La mondialisation de l'économie, le rôle hégémonique de l'informatique, le pouvoir d'une économie dématérialisée, les nouvelles formes d'autoritarisme liées au contrôle de la communication, tous ces thèmes paraissent laisser indifférents les écrivains de la " grande littérature ", du moins en Europe. Dans la plupart de leurs romans, le monde semble immuable. Dominent les histoires intimistes, qui auraient pu se passer il y a cinquante ans - ou qui pourraient se produire dans cinquante ans... Amours, passions et trahisons perpétuent leur consommation sous une lumière tamisée, dans un monde aux couleurs pâles et aux fragrances de poussière et de talc. Certes, il y a quelques exceptions ; mais, la plupart du temps, le cadre général est immodérément " minimaliste » ". Valerio Evangelisti, " Une littérature des « étages inférieurs »... ", art. cit.

9. À ceux où le nom d'Eymerich figure dans le titre, ajouter Cherudek, op. cit., ainsi que Picatrix... op. cit. et Mater Terribilis, op. cit.

10. Metallo urlante, op. cit. 
dominicain, Inquisiteur général du royaume d'Aragon, auteur d'un manuel à l'usage de ses pairs et disciples, fort diffusé et mis en pratique pendant des décennies), préfere parvenir à ses fins par la pression psychologique, la ruse, si ce n'est le parjure pieusement légitimé, plutôt que par l'exercice de la violence physique, même si la torture fait partie de son arsenal et s'il fait fort bon marché de la vie humaine, "pour la plus grande gloire de Dieu, c'est-à-dire de l'Église ». Cela renvoie sans doute à la violence du pouvoir, mais avec une forte ambiguïté, ou une forte ambivalence. Eymerich est en effet tout à la fois odieux et fascinant, et son statut même de héros récurrent, renforcé par sa stature intellectuelle, amène le lecteur à s'identifier à lui, tout en ne pouvant pas, du moins on l'espère, ne pas se demander à un moment donné ce qui se passe, et comment il en arrive à approuver un tel personnage - c'est du moins l'intention explicite d'Evangelisti ${ }^{11}$. En même temps, on peut lire dans ces textes comme le résultat et le résumé d'une évolution de la figure du juge aux yeux d'une partie de l'opinion italienne qui, entre les anni di piombo et les conséquences de l'opération mani pulite, a pu prendre partie à la fois contre les juges qui ont condamné sans preuve de supposés terroristes ${ }^{12}$, et pour ceux dont l'action a fait éclater un système de corruption (juges qu' il y a peu, tel chef de gouvernement de rencontre se permettait de couvrir de basses invectives à prétentions anthropologiques).

Passons du personnage à son auteur puis à la façon dont ce dernier utilise sa création : Valerio Evangelisti, Bolonais, né en 1952, est historien de formation. Il a gagné sa vie en travaillant pour l'administration du cadastre, et a publié, avant ses romans, divers ouvrages consacrés aux socialistes italiens dans les années 1880, aux luttes sociales en ÉmilieRomagne dans la même période, aux "gauches hérétiques " (concept englobant tout à la fois la Bande à Bonnot et les sandinistes), aux jacobins de Bologne entre 1792 et 1797 , aux punks et aux contras ${ }^{13}$, plus une quarantaine d'articles, dans diverses revues ${ }^{14}$ et ouvrages collectifs (par

11. "Cela m'amuse beaucoup d'avoir un héros très méchant et de voir des gens qui s’identifient à lui et ensuite se demandent ce qui leur est arrivé. Je fais quelque chose de diabolique avec mes lecteurs, je les oblige à être mauvais, vraiment très mauvais, en espérant qu’à la fin ils se demandent de qui il s'agit, et qu'ils se rendent compte que c'est bien d'eux ". Interview de Valerio Evangelisti, Galaxies, revue citée.

12. "Valerio Evangelisti sull'arresto Persichetti », 29 août 2002, Internet, "Indymedia Vicenza ".

13. Valerio Evangelisti et Emanuela Zucchini, Storia del Partito socialista rivoluzionario 1881-1893, Bologne, Cappelli 1981 ; Valerio Evangelisti et Salvatore Secchi, Il galletto rosso. Precariato e conflitto di classe in Emilia Romagna 1880-1890, Venise, Marsilio, 1982 ; Valerio Evangelisti, Sinistre eretiche Dalla banda Bonnot al sandinismo, 1905-1984, Milan, SugarCo, 1985; id., Gallerie nel Presente; Punks, Snuffs, Contras : tre studi di storia simultanea, Lacaita, 1988 ; id., Gli sbirri alla lanterna. La plebe giacobina bolognese dall'anno I all'anno V(1792-1797), Bologne, Bold Machine 1991.

14. Il Mulino, Rivista di storia contemporanea, Quaderni sardi di storia, Quaderni emiliani, etc. 
exemple un essai sur l'Automne chaud de $1969{ }^{15}$ ). Il a aussi dirigé de l'extrême fin des années 1980 à 1996 une revue, Progetto memoria, "Rivista di Storia dell'Antagonismo Sociale », et y a donné entre autres des textes sur les premières années du FPLP, sur la première guerre du Golfe ou sur Wilhelm Reich. Dans le même temps, ayant abandonné, aux alentours de 1988, tout espoir de titularisation dans l'université italienne, il s'est essayé au roman d'horreur, en partie par admiration pour les films de la Hammer, et a cherché un personnage convenablement effrayant, moins usé que les vampires, loups-garous et autres momies, mais tout aussi apte à focaliser les sympathies. D'où l'inquisiteur Nicola Eymerich, à l'état de fantôme dans un roman d'épouvante resté inédit et désavoué, puis rendu à son XIV siècle d'origine (quitte à affirmer qu'Umberto Eco et le Nom de la Rose n'ont rien eu à voir dans ce choix) et mêlé à des romans placés, fond et forme, sous le signe du mélange : l'auteur joue en effet avec les codes du roman historique, promenant son inquisiteur espagnol au Val d'Aoste, dans le Frioul, en Sardaigne, en Occitanie, en Andalousie ou aux Canaries ; il joue aussi avec les codes de la science-fiction, entre univers parallèles et communiquant les uns avec les autres, manipulations génétiques ou recyclage de théories scientifiques fantaisistes (à commencer par celles de Wilhem Reich, déjà cité, sur les « orgones » et le cancer)... Il joue enfin, et peut-être surtout, avec les codes du fantastique ou de la fantasy, en confrontant son personnage à des phénomènes inexpliqués, monstrueux ou aberrants - au moins du point de vue de l'inquisiteur -, en particulier des cultes féminins renvoyant à Diane, Hécate, etc. et produisant des effets réels. C'est là, en fait, l'élément dominant, avec une sorte d'inversion d'un sous-genre connu, le « fantastique rationalisé » : ici, les explications rationnelles, ou supposées telles, qu'elles fassent intervenir les intrigues politiques, la technologie (métaux à mémoire des formes par exemple) ou des hallucinogènes naturels type ergot de seigle, finissent toujours par achopper, par se heurter à un noyau irréductible, que les forces surnaturelles supposées à l'œuvre aient à voir avec les dieux de l'antiquité, avec le Vaudou et "Ogou ferraille », ou avec des entités plus ou moins héritées d'Howard Phillips Lovecraft. Par ailleurs, on a affaire à une mythologie générale, qui oppose l'Église aux cultes déjà cités, la raison à la sensibilité, mais aussi - et on finit par se rapprocher du sujet - des forces guerrières, métalliques, strictement masculines, d'un côté, à d'autres, liées à ces cultes féminins, à la vie, à une humanité complète. Ce qui renvoie

15. Valerio Evangelisti et Salvatore Secchi, "L'autumno caldo : l'offensiva della classe operaia ", p. 335-354 de Storia della società italiana, 24, Il miracolo economico e il centro-sinistra, Milan, Teti, 1990. 
d'ailleurs au personnage même d'Eymerich, calqué sur le tableau clinique de la personnalité schizoïde, ainsi que sur les inquiétudes d'Evangelisti à propos de sa propre part d'ombre, et qui, au fil de la publication des romans, se trouve de façon de plus en plus précise face à tout ce qu'il entend refouler : la confrontation avec le féminin, refusée, imposée, nécessaire et constitutive d'une normalité minimale, est d'ailleurs un thème majeur, permanent, à la fois dans les volumes mettant Eymerich en scène et dans l'ensemble sinon des textes, du moins des romans d'Evangelisti (les nouvelles, entre brièveté et caractère parfois circonstanciel, pouvant être mises à part).

Au mélange entre littératures de genre s'en ajoute un autre, celui de ces dernières, revendiquées, et de la littérature "légitime ", du moins si l'on considère avec Umberto Eco que le roman populaire est consolateur alors que la Littérature "place le lecteur en guerre contre lui-même " ce qui correspond à une volonté explicite d'Evangelisti ${ }^{16}$ (encore qu'on puisse plaider que ce sentiment de guerre contre soi-même puisse être consolateur au second degré, en donnant la rassurante et satisfaisante sensation de consommer de la "vraie " culture à peut-être moindres frais - ce serait alors un nouveau visage, plus élaboré, de la midcult ${ }^{17}$ ).

À ces premiers mélanges s'en surajoute un autre, formel, lié à une ficelle classique du roman populaire : la narration en parallèle, qui permet d'accentuer les suspenses en alternant deux récits ou plus. En gros, l'un, le principal, met en scène Eymerich en son temps, tandis que l'autre, ou les autres, situé(s) ailleurs et dans d'autres époques, à commencer par notre passé immédiat et notre futur proche, lui est ou lui sont lié(s) directement ou indirectement, et d'une façon qui n'apparaît souvent qu'à la fin du roman. Ils renvoient en revanche à la science-fiction, ou plutôt à une politique-fiction fort noire. Si le mélange est plus limité pour les trois volumes d'une biographie imaginaire de Nostradamus (œuvre de commande où seule l'époque du héros ou anti-héros éponyme pouvait être prise en compte), on n'y retrouve pas moins, mêlé au roman historique jouant sur l'accumulation des personnages connus - de Rabelais au cardinal Jean Du Bellay -, l'affrontement d'entités, une lutte mythologique entre principes,

16. "Je crois beaucoup à la science-fiction et aux littératures de l'imaginaire en général comme littérature de l'inquiétude. Je ne veux pas apporter de consolation dans mes livres, mes histoires sont souvent complètement amorales, il y a des personnages qui n'ont pas une position exacte entre le bien et le mal. Je veux troubler le lecteur pour le soustraire aux certitudes qu'on lui impose dans ce domaine ». Interview de Valerio Evangelisti, Galaxies, revue citée.

17. Umberto Eco note que " à notre époque, on aura un roman populaire même là où le héros se révélera problématique de façon prévisible». De Superman..., op. cit., p. 19. 
parallèle cosmique ou cosmogonique d'une évolution du Michel de Nostredame romanesque dans ses rapports sinon avec "La Femme ", du moins avec les femmes. Enfin, on retrouve tous les éléments déjà cités dans ce qui semble constituer une nouvelle série, autour du personnage principal d'une nouvelle, puis de deux romans, sorcier et pistolero dans l'Amérique de la guerre de Sécession, tandis que dans un futur cette fois lointain, l'an $3000^{18}$, la planète Terre est devenue un gigantesque asile de fous qui s'entre-massacrent. On pourrait d'ailleurs s'interroger sur l'opposition entre cette continuité permanente reliant les époques et la rupture opposant l'histoire et le présent selon Pier Paolo Pasolini.

Dans chaque roman, les histoires mêlées, et en résonance les unes avec les autres, accordent une large place à la violence, même s'il s'agit, dans le cas d'Eymerich, d'une violence froide, appliquée à froid aux autres voire à lui-même lorsqu'il s'agit de brûlures volontaires, superficielles, destinées à modifier son aspect physique et à le faire passer pour un autre, dans la pure tradition du feuilleton du XIX ${ }^{e}$ siècle... Mais le plus significatif n'est sans doute pas cela, mais plutôt le contexte. En effet, on y trouve, omniprésente, la guerre, et non pas n'importe quel conflit, mais un affrontement inégal, qui n'oppose pas État à État - ou armée à armée, comme ce serait le cas pour la guerre civile espagnole. Et la guerre de Sécession déjà évoquée n'est en rien un contre-exemple, car elle n'est pas vue comme le terrible laboratoire de la guerre moderne, l'avant-goût de la Première Guerre mondiale qu'elle a été, mais comme une simple toile de fond pour les sinistres exploits de soldats de fortune, de petits groupes de tueurs, relevant - pour ceux que l'on suit - de la légende du loup-garou, groupés non pas autour du drapeau sudiste pour lequel ils sont censés se battre mais sous le drapeau noir donnant son titre à Black Flag. On les voit moins livrer bataille - sauf de façon brève et désastreuse - qu'assassiner des civils ou des prisonniers avant d'envisager d'aller par exemple au Mexique pour se mettre au service de Maximilien contre Juarez. Dans la biographie imaginaire de Nostradamus, sans même que l'auteur, à l'en croire, l'ait prévu au départ, les guerres de religion se sont fort vite retrouvées au premier plan, avec en particulier le massacre des vaudois du Luberon - on est toujours dans le domaine des massacres de civils par une soldatesque. Les guerres au cours desquelles on retrouve Eymerich, elles, sont des opéra-

18. En partie au moins du fait de la commande, pour une anthologie française, d'une nouvelle ensuite intégrée à ce roman, ( Paradi ", Robert Silverberg et Jacques Chambon (dir.), Destination 3001, Paris, Flammarion, 2000). À noter toutefois que ce futur lointain est lié de manière explicite à des psychopathies présentes, en l'occurence à un « serial killer » dont le cas a été longuement analysé (cf. Valerio Evangelisti, "American psychosis ", 1998, rééd. in Alla periferia..., op. cit.). 
tions de politique intérieure ou des épisodes de guerre civile : par exemple l'expédition de 1354 menée par Pierre IV le Cérémonieux, roi d'Aragon, pour réduire la révolte d'Arborea en Sardaigne, ou, en 1369, à Montiel, la dernière bataille perdue par Pierre le Cruel, roi de Castille, contre son demi-frère Henri de Trastamare, allié de la papauté, et contre ses mercenaires commandés par Bertrand du Guesclin. De façon significative, dans ce dernier cas, Eymerich circule entre l'extérieur et l'intérieur du château où le roi est encerclé, et s'il agit contre lui c'est avec des objectifs bien plus vastes de son point de vue que la préparation d'une succession précipitée au trône. De même, la guerre de Cent ans peut fournir une toile de fond, l'inquisiteur peut participer en 1360 à la préparation du traité de Brétigny (la libération de Jean II le Bon contre rançon et cession de territoires) mais il a affaire à des soldats perdus, débandés (qui se battent pour leur propre compte ou relèvent du thème fantastique traditionnel du zombie), et surtout à une secte sanglante... Bien entendu, ces choix renvoient aux réalités du temps quant à la nature de la guerre, mais ils n'en sont pas moins toujours des choix, correspondent à un point de vue, et on est tenté de renvoyer à l'aphorisme crocien selon lequel toute histoire est histoire contemporaine, c'est-à-dire parle du moment où elle est écrite (principe a priori difficile à contourner pour un historien engagé comme l'a été Evangelisti). D’ailleurs, malgré les apparences, malgré par exemple un souci d'effets stylistiques simples (phrases plus longues, peut-être baroques, quand il est question de l'époque d'Eymerich, plus courtes et sèches pour le présent et le futur), il ne s'agit pas dans ces romans de reconstitution archéologique, l'anachronisme n'est pas traqué de manière très sourcilleuse. Ainsi, des personnages du XIv siècle peuvent parler d'amibes, découvertes au XVII ${ }^{\mathrm{e}}$, et cette erreur ne saurait avoir aucun caractère de gravité. Il s'agit bien de parler de violences présentes bien plus que de violences passées.

En ce qui concerne si ce n'est ce présent stricto sensu, du moins l'époque que la corporation des historiens qualifie de contemporaine, on pourra peut-être considérer comme significative l'absence des guerres napoléoniennes, du Risorgimento, ou de la Première Guerre mondiale. Peut-être cette absence s'explique-t-elle simplement par une réaction contre les ponts aux ânes de l'histoire nationale, par un refus conscient de se centrer sur l'Italie, de traiter d'une violence qui serait spécifique à ce pays. Cela dit, rien n'obligerait - au moins dans deux cas sur trois - à s'enfermer dans une perspective nationale, et des relectures historiographiques assez récentes offriraient d'intéressantes perspectives, surtout pour ce qui est de la "Grande Guerre ». Quant à la Seconde Guerre mondiale, ce qui en est 
envisagé, de façon d'ailleurs conforme à une réalité terrifiante et incontournable, ce sont les camps d'extermination, et plus précisément les expériences délirantes et monstrueuses menées par de prétendus scientifiques, entre proto-manipulations génétiques et bricolages renouvelés du docteur Frankenstein, inscrites dans une sinistre continuité. Disons qu'il est sans doute (et en tant que citoyen on ne le reprochera certes pas à l'auteur) moins question de la guerre que du nazisme, du racisme, de l'antisémitisme, en fonction d'ailleurs d'inquiétudes actuelles ${ }^{19}$, avec des échos très clairs tant pour l'époque d'Eymerich que pour la suite, pour notre présent le plus immédiat ou pour un avenir proche fort peu réconfortant. On tient sans doute là un élément d'explication quant à la volonté délibérée d'éviter l'histoire italienne stricto sensu, non parce que c'est celle du pays de l'auteur, mais parce que c'est celle d'un Etat-nation : Evangelisti enjambe l'ère de ces États-nations, avec l'idée qu'ils ne sont plus d'actualité désormais. Il les évite même lorsqu'il relit le passé proche : ainsi, la guerre d'Algérie est évoquée sous l'angle de réseaux d'extrême droite et de nouveau - du racisme, et non sous celui d'une guerre d'indépendance, ce qui ne s'explique pas seulement par la distance que le temps a permis de prendre avec un FLN fort différent des images et légendes tiers-mondistes : la guerre, ici, est directement associée aux exactions du Ku Klux Klan, mais aussi à l'assassinat de Kennedy, etc. Pour l'avenir, tel qu'il se dessine pièce par pièce - mais de façon cohérente - dans les différents romans, Evangelisti met à mal les nations constituées en imaginant à la fois un éclatement des États-Unis et un affrontement en Europe et en Afrique entre deux entités transnationales. Des épidémies, conséquences dévastatrices de phénomènes dont on suppose qu’ils ont été déclenchés au temps d'Eymerich, coupent les États-Unis en trois États : un que l'on pourrait qualifier de new-age et pseudo-orientalisant, un autre, de néolibéral et d'ultra-monétariste, le troisième, de fondamentaliste et de raciste. Ils ne s'opposent pas entre eux et ont en particulier des façons assez équivalentes de traiter leur jeunesse et de déporter leurs dissidents dans la même île lointaine - en fait, la Sardaigne. La guerre, évoquée ou en partie racontée, est alors une guerre civile, notamment celle de l'État " sudiste " contre les Noirs. En parallèle, de volume en volume, on voit sévir une organisation, la RACHE ("vengeance " en allemand), héritage nazi, d'abord caché sous diverses apparences, dont celle d'une Église ou d'une secte, puis s'emparant dans un avenir proche des Balkans et jusque

19. Cf. Valerio Evangelisti, «L'extrême droite... ", art. cit. 
du nord-est de l'Italie, récupérant enfin les expériences monstrueuses déjà citées, et d'autres conduites sous Elena Ceaucescu, pour créer des soldats biologiques artificiels. Cette RACHE a de nets aspects néo-féodaux. Elle met en avant de pseudo-aristocraties autour de mythes fondés sur le fer, le sang, le feu, et est combattue plutôt mollement par un "Eurocorps ", expression moins d'une Europe politique que d'une « Eurobank». Celle-ci a de fait abandonné des territoires à ses ennemis et, même si elle est plus " humaine " qu'eux, elle leur semble assez symétrique, ne serait-ce que dans l'emploi de soldats artificiels, différents, mais eux aussi issus d'expériences nazies, plutôt frankensteiniennes en l'occurrence. L'aboutissement de tout cela pourrait être le gigantesque asile de fous de l'an 3000 déjà évoqué.

On a ainsi affaire à une brutalité protéifoirme, anomique, d'origine plus psychologique que sociale ou politique, la guerre n'ayant pas un statut distinct de celui d'autres formes de violence, cristallisées par Eymerich et par l'Inquisition (la nature des bûchers d'autrefois n'est pas différente de celle des guerres à venir). Violence publique et violences privées s'imbriquent étroitement à la fois dans les réalités de l'époque médiévale, dans celles de guerres civiles, de guerrillas, de guerres qui n'opposent pas, ou plutôt n'opposent plus, des entités étatiques, et bien entendu dans la réalité des souffrances subies. On assiste à un effacement des limites entre différentes catégories de violence, et, semble-t-il, à un refus de les distinguer. On a aussi, et sans doute surtout, un retour à une barbarie, au-delà de rêves humanitaires de régulation de la guerre et même de son industrialisation, de sa mécanisation, phénomènes propres au second XIx ${ }^{\mathrm{e}}$ siècle et à une bonne partie du Xxe . Cela pourrait rejoindre des considérations d'actualité, comme celles du général Fabio Mini, qui évoque en 2003 une marchandisation de la guerre, une régression de plusieurs siècles dans son organisation, une concaténation entre crime organisé et mercenariat, etc. ${ }^{20} \mathrm{Il}$ y aurait là de quoi alimenter le discours sur la capacité des littéra-

20. "Molti interventi armati di questi ultimi anni hanno avuto come principali beneficiari non gli Stati stessi ma [...] le singole corporazioni » [...]. Oggi la guerra è [...] "possibile» soltanto come manifestazione di un ritorno all'ordinamento confessionale. Laddove la nuova 'confessione' è il mercato. Un passo indietro di cinque secoli nell' ordinamento giuridico della guerra e uno di dieci per la componente di fanatismo che tale ordinamento comporta [...] Le compagnie private stanno poi assumendo per conto dei militari (e ovviamente di tutte le organizzazioni committenti) anche veri e propri compiti operativi che una volta non era assolutamente immaginabile fossero attribuiti a dei civili. Compagnie private fanno la guardia a istallazioni militari anche nei teatri operativi, gestiscono le comunicazioni, forniscono intelligence specializzata, effettuano attività di sminamento, sorveglianza aerea del campo di battaglia e così via. Basta pagare [...] La realtà è che l'impero della guerra di questo millennio, sottoposto alle spinte dell' impero del terrore, del crimine, dell'economia e di quant' altro, è concettualmente e intellettualmente regredito. Si ritrova in una fase primitiva in cui misura la propria efficacia dalla potenza e dalla distruzione che riesce a esprimere. Non è in grado di calibrare l'uso della forza sugli scopi 
tures de genre à appréhender le réel immédiat, et à en rendre compte plus efficacement que les formes supposées "légitimes".

Il faudrait sans doute souligner et développer d'autres aspects de la violence telle qu'elle est décrite ou mise en scène par Valerio Evangelisti, et en particulier deux d'entre eux, fort évidents. D'abord, et même si cela semble contredire ce qui vient d'être affirmé quant à l'appréhension du réel, les violences concrètes de la société italienne contemporaine ne sont pas envisagées, du moins en apparence. Ensuite, l'ambiguïté est permanente quant au jugement porté sur l'exercice même de la violence. Bien entendu, l'absence de faits concrets immédiats renvoie au parti pris de décalage chronologique et géographique indiqué plus haut, mais on peut soupçonner un fonctionnement inverse des liens de causalité. Cela pourrait sembler étrange, compte tenu des intérêts manifestés par Valerio Evangelisti en tant qu'historien, mais aussi de ses liens amicaux, par exemple avec l'auteur de romans policiers Cesare Battisti, ou de ses interventions publiques, comme lors de l'arrestation en France de Paolo Persichetti ${ }^{21}$. On peut cependnnt imaginer une difficulté particulière à rendre compte des événements vécus, peut-être parce qu'ils ont débouché sur une défaite, mais aussi parce que, dans le cadre choisi - un roman populaire ne pouvant qu'être simplificateur -, il serait trop compliqué de rendre compte des raisons de cette défaite, des erreurs commises, etc. Ainsi, le discours de la révolte, revendiqué, passe par des masques, des décalages, des transpositions. D'autant que les difficultés immédiates inhérentes à un tel discours coexistent avec l'impression qu'il est fort difficile de le tenir dans une Italie d'où il est supposé avoir été éradiqué, que ce soit là une réalité ou un fantasme lié, de nouveau, à la défaite ${ }^{22}$. Dans les deux cas, on pourrait d'ailleurs évoquer stricto sensu une forme de littérature de reflux, ou plutôt adaptée à un reflux. Cette idée d'une adaptation défensive est corroborée par un thème facile à mettre en rapport avec la situation de l'Italie et en particulier avec les caractéristiques concrètes

da ottenere, così come non riesce a immaginare un dopoguerra che faccia parte del processo della guerra stessa al punto di dettarne le condizioni ». Fabio Mini, La guerra dopo la guerra. Soldati, burocrati e mercenari nell'epoca della pace virtuale, Turin, Einaudi, 2003, p. 29, 126, 290. Cf. aussi p. 118-135.

21. Cf. "Valerio Evangelisti sull'arresto Persichetti » art. cit.

22. «Mais ce n'est pas seulement une question de Berlusconi. En Italie, pour effacer même le souvenir des luttes sociales des années 70 , on a fait table rase de la mémoire. Toutes les disciplines universitaires ont été aplaties, le révisionnisme historique a eu la place d'honneur, même dans sa version négationniste. Les journaux et les chaînes de télé se sont chargés de rendre capillaire l'opération. Le journaliste, au moins dans mon pays, a pris la place de l'intellectuel, mais avec une mission inverse : faire oublier que les phénomènes ont des causes. Les consciences se sont vidées, substituées par des séries d'images sans rapport réciproque ». Intervention de Valerio Evangelisti sur le site Internet de l'émission " Mauvais genres » (France Culture). 
de son paysage audio-visuel, même si on trouverait à l'appuyer sur la réalité d'autres pays : la " colonisation de l'imaginaire ", théorisée ${ }^{23}$, mais aussi mise en scène dans un avenir fictif, avec un système de contrôle des rêves et de l'inconscient collectif, d'ailleurs condamné à l'échec ${ }^{24}$. Ainsi, le souvenir des années de plomb, celui des " années de boue ${ }^{25}$ ", leurs conséquences pérennes à base de cleptocratie cathodique, puis, qu'on les partage ou non, les réactions aux campagnes d'opinion liées aux deux guerres du Golfe, se retrouvent dans des textes qui font mine de ne pas en parler, ou du moins n'en parlent pas de façon directe. On peut d'ailleurs imaginer une évolution future et un discours plus explicite, en extrapolant à partir du dernier volume en date, Antracite, inclus dans le cycle lié à la guerre de Sécession, et où il est notamment question des premières organisations syndicales dans les mines américaines. On aurait ainsi, même si c'est toujours de manière indirecte, un retour à un discours sur la violence sociale, alors même que celle-ci, après une période de très forte présence, semble à peu près absente de la littérature italienne récente, qu'elle soit « légitime» ou "de genre».

Le second aspect évoqué plus haut pourrait contribuer à expliquer ce silence, si l'on suppose que, tant la condamnation que la fascination, entre lesquels oscillent les textes à propos de la violence, poseraient des problèmes si elles s'appliquaient à l'histoire récente de l'Italie. Cette oscillation est évidente autour du personnage même d'Eymerich, héros répulsif, mais aussi de Nostradamus, qui n'a rien du personnage sympathique à l'origine, et qui se construit petit à petit, ou de personnages secondaires comme tel soldat d'un néo-Ku Klux Klan, "beauf ", sans doute fasciste,

23. "Colonizzare l'immaginario. Sembrava impossibile, eppure basta disporre degli strumenti opportuni. Televisioni, mass-media, una stampa docile, un trend culturale. Finisce che intere generazioni si trovano immerse in un sogno, e lo scambiano per realtà. Ora, quali sono le caratteristiche di un sogno ? Che si vive una vicenda priva di antecedenti e di conseguenze nel futuro. Esiste il presente e basta ». Valerio Evangelisti, «... et mourir de plaisir ", éditorial, Carmilla, n 1 , 1995. " Le capitalisme traditionnel se contentait de la publicité. Désormais, il va plus loin : dans l'imagination, dans les rêves, dans les visions du monde les plus intimes. La croissance de la communication le lui a permis, en imposant des modes de vie, en créant des besoins là où il n'y en avait pas, en augmentant la soif d'affirmation de l'individu. On ne comprend rien à la société contemporaine si l'on ne tient pas compte de la rapide colonisation de l'imaginaire accomplie ces dernières années. Auparavant, on jouait un rôle productif un certain nombre d'heures par jour, le reste du temps étant consacré au divertissement et au repos, c'est-àdire à soi-même. Les activités de détente, toutes fondées sur la communication, étendent le champ de la productivité aux dépens du loisir et du temps de repos. Presque tous les spectacles télévisuels contiennent des incitations à l'achat, qu'il s'agisse de publicité explicite ou de références à des modes de vie considérés comme les meilleurs pour tous ". Valerio Evangelisti, "Une littérature des " étages inférieurs "... ", art. cit.

24. "La fuite de la couveuse ", nouvelle citée. La référence à la guerre du Golfe, à travers Saddam Hussein, y est explicite.

25. Cf. Indro Montanelli, Mario Cervi, L'Italia degli anni di fango (1978-1993), Milan, Rizzoli, 1993. 
mais au bout du compte humain, face aux chefs extrémistes doctrinaires de son camp, représentants d'une déshumanisation radicale ${ }^{26}$. Cela correspond à la volonté d'effacer les barrières du manichéisme, de mêler le bon et le mauvais, d'autant que les armées noires que le camp affronte ne valent guère mieux que lui : la violence impose ses règles au-delà des causes défendues, et les victimes, ou les perdants, ne sont pas automatiquement sympathiques. Cette règle ne disparaît que pour des guérilleros sud-américains, magnifiés ou édulcorés peut-être au-delà du raisonnable, dans des nouvelles, il est vrai, non reliées aux cycles principaux : les FarC colombiennes liquident un Président américain sans autre effusion de sang, et d'une façon trop pornographique pour soulever la réprobation (un émule de Bill Clinton est puni, disons, par là ou il a péché) ; les Péruviens du mouvement insurrectionnel Tupac Amaru sont soucieux de préserver toute vie, même animale ${ }^{27}$, etc.

Reste qu'en dehors de ces nouvelles, qu'une fois encore on supposera assez marginales, il me semble que le discours sur la guerre et la violence, au long des romans, est caractérisé par une volonté de brouiller les repères du lecteur, surtout par une évacuation des Etats, ce qui débouche aussi sur une vision du monde où la guerre, tout à fait réelle et dévastatrice, est pourtant avant tout intérieure, métaphysique ou psychologique, si l'on veut. C'est la lutte mythologique entre principes, entre Hélios ou Mars et Séléné ou Diane, entre un univers de la guerre, du métal, de la mathématique et aussi de l'Église, supposé purement masculin, hostile au corps et à la vie, et un univers complet, fusionnel dans doute, magique en partie, même si l'ironie sur le new-age suppose que l'auteur ne soit pas tout à fait dupe... D'une certaine façon, au-delà des guerres décrites et rêvées, des violences, le combat fondamental est bien celui mené contre les tendances schizoïdes, contre les hémiplégies de l'âme considérées comme la source de ces guerres et de ces violences. Ce qui fait chercher, au-delà cette fois de la littérature populaire et des engagements, un discours d'Evangelisti sur l'humanité et sur lui-même, sur le général et sur le singulier, discours facilité et en même temps camouflé par les normes et les ficelles des littératures de l'imaginaire, discours discutable aussi : mais il me semblait inté-

26. "Même mes fascistes, comme Robinson dans Metallica [nouvelle reprise dans Metallo urlante, op. cit.], ne sont pas entièrement mauvais. En fait, c'est l'Histoire qu'il faut juger, et pas eux. Ce qui est en action dans mes livres, c'est la force des choses, comprise dans un sens très matériel. Ce que fait Eymerich, en particulier, est justifié par la mission qu'il doit accomplir, et qui est une mission historique, les hérétiques ne sont pas meilleurs que lui, peut-être au contraire est-il le meilleur, puisqu'il voit très loin... » Interview de Valerio Evangelisti, Galaxies, revue citée.

27. "Il nodo Kappa » et " Le souffle des FARC ", nouvelles citées. 
ressant d'essayer de le défricher, même si c'est de façon sommaire et confuse, en vue, je l'ai dit, d'éventuelles comparaisons du côté d'une littérature " légitime ", à laquelle il s'agit peut-être de disputer la représentation du réel, en s'éloignant apparemment de ce dernier spour mieux s'en rapprocher. 